Katiuska Reynaldos

Grandón ${ }^{1}$

Javiera Achondo Oisel ${ }^{2}$

Valentina Azolas

Valenzuela ${ }^{2}$

${ }^{1}$ Enfermera-matrona. Ingeniero Comercial. Magíster en Administración de Empresas-MBA. Doctor en Ciencias Empresariales. Profesor Asociado, Facultad de Enfermería Universidad Andrés Bello, Santiago, Chile.

Autor de Correspondencia: katiuska.reynaldos@unab.cl Licenciada en Enfermería,

Facultad de Enfermería

Universidad Andrés Bello, Santiago, Chile

Trabajo recibido: $01 \mathrm{de}$ Agosto de 2017. Aprobado: 28 de Agosto de 2017.

\section{FACTORES INFLUYENTES EN LA SATISFACCIÓN USUARIA DE LA ATENCIÓN PRIMARIA EN SALUD LATINOAMERICANA: REVISIÓN INTEGRADORA.}

FACTORS THAT INFLUENCE THE USER SATISFACTION OF PRIMARY HEALTH CARE LATIN AMERICAN: INTEGRATIVE REVIEW.

FATORES INFLUENTES NA SATISFAÇÃO DO USUÁRIO DA ATENÇÃO PRIMÁRIA NA SAÚDE DA AMÉRICA LATINA: REVISÃO INTEGRATIVA.

\section{Resumen}

Introducción: La satisfacción usuaria se comprende como el grado de congruencia que existe entre las expectativas del usuario de una atención ideal en salud y la percepción de éste del servicio que recibió.

Objetivo: Determinar los factores que influyen en la satisfacción usuaria de la atención primaria de salud en Latinoamérica entre los años 2000-2016.

Método: Revisión bibliográfica realizada en los meses de Octubre -Noviembre del año 2016, de artículos relacionados con la satisfacción usuaria en atención primaria de salud obtenidos a través de booleanos y limitantes como el periodo de publicación, idioma español y textos completos, de los cuales se escogieron 12 de ellos.

Resultados: Los estudios planteados fueron revisados según los objetivos, factores influyentes en la satisfacción usuaria, resultados y sugerencias de plan de mejora en el primer nivel de atención de salud. El análisis considera mayoritariamente países como Chile y Colombia, incluyendo también México, Perú y El Salvador. 
Conclusiones: Se hace esencial impulsar el desarrollo de nuevos estudios que integren los factores influyentes en la satisfacción usuaria, no como un método de evaluación en sí, sino como, un instrumento para crear las bases de mejoras en la atención de forma efectiva y considerando las necesidades de la población, con el objetivo de dar soluciones tanto a las expectativas como necesidades reales de los pacientes/usuarios.

Palabras claves: Satisfacción del Paciente, calidad de atención de salud, atención primaria salud.

\section{Abstract}

Introduction: Customer satisfaction is understood as the degree of congruence that exists between the expectations of the user of an ideal health care and the perception of the service received.

Objective: To determine the factors that influence in the satisfaction of primary health care Latin American users, between 2000 and 2016.

Method:Bibliographical review carried out on October and November 2016, of articles related to user satisfaction in primary health care, obtained through booleans, and limitations such as the publication period, Spanish language and completes texts, in which 12 of ones are selected.

Results: The studies contemplated were reviewed according to the objectives, factors influencing in user satisfaction, results and suggestions for improvement the plan in the first level of health care. The analysis considers chiefly countries like Chile and Colombia, including also Mexico, Peru and El Salvador.

Conclusions: Becomes essential to stimulate the development of new studies that it integrate the factors influencing user satisfaction, not as an evaluation method per se, but as an instrument to create the bases for improvements in care effectively and considering the needs of the population, with the aim of providing solutions both to the expectations and real needs of patients / users.

Keywords: Patient Satisfaction, Quality of Health Care, Primary Health Care

\section{Resumo:}

Introdução: a satisfação do usuário é entendida como o grau de congruência que existe entre as expectativas do usuário de um cuidado de saúde ideal e a percepção do serviço recebido.

Objetivo: Determinar os fatores que influenciam a satisfação dos usuários de cuidados de saúde primários na América Latina entre os anos 2000-2016.

Método: revisão bibliográfica realizada nos meses de outubro a novembro de 2016, de artigos relacionados à satisfação dos usuários em cuidados de saúde primários obtidos através de booleanos e limitações como o período de publicação, língua espanhola e textos completos, dos quais 12 foram escolhidos.

Resultados: os estudos foram revisados de acordo com os objetivos, fatores que influenciam a satisfação dos usuários, resultados e sugestões para o plano de melhoria no primeiro nível de cuidados de saúde. A análise considera principalmente países como Chile e Colômbia, incluindo também México, Peru e El Salvador.

Conclusões: é essencial promover o desenvolvimento de novos estudos que integrem fatores que influenciam a satisfação do usuário, não como um método de avaliação per se, mas como um instrumento para criar bases de melhorias de atendimento efetivamente e considerando a necessidades da população, com o objetivo de fornecer soluções tanto para as expectativas quanto para as necessidades reais dos pacientes / usuários. 
Palavras chaves: Satisfação do paciente, qualidade dos cuidados de saúde, cuidados de saúde primários

\section{Introducción}

La Atención Primaria de Salud (APS) es un componente fundamental de un sistema de salud efectivo, ya que, es una estrategia para fortalecer la capacidad de la sociedad y reducir las inequidades en salud ${ }^{(1)}$. Esta es definida por la Organización Mundial para la Salud (OMS) como: "La asistencia sanitaria esencial accesible a todos los individuos y familias de la comunidad a través de medios aceptables para ellos, con su plena participación y a un costo asequible para la comunidad y el país" (2).

Los Centros de Salud Familiar (CESFAM) creados en el año 1997 basan su atención en el denominado "Modelo de Atención con Enfoque Familiar en la Atención Primaria de Salud". Tienen el fin de presentar al usuario equipos de trabajo que ofrezcan atención personalizada y continuada, con énfasis en aspectos preventivos e incluyendo la fuerte participación de la comunidad ${ }^{(3,4)}$.

Esta estrecha relación con la comunidad que sugiere este modelo de atención, es lo que se ve perjudicado cuando el usuario no está satisfecho con la atención de la institución influyendo negativamente en el proceso. Por consiguiente, la satisfacción del usuario es definida como: "el grado de congruencia que existe entre las expectativas del usuario de una atención ideal en salud y la percepción de éste del servicio que recibió, o bien, como la medida en que los profesionales de salud logran cumplir las necesidades y expectativas del usuario" ${ }^{(5)}$. Es por tal motivo que surge la necesidad de conocer qué factores influyen en la satisfacción usuaria del primer nivel de atención de salud en Latinoamérica.

Esta revisión bibliográfica tuvo por objetivo determinar los factores que influyen en la satisfacción usuaria de la atención primaria de salud en Latinoamérica entre los años 20002016. Dicha revisión permite identificar las necesidades de los usuarios y así generar bases que permitan una mejora continua en la atención del primer nivel de salud. La búsqueda fue realizada en bases de datos como recursos electrónicos con búsqueda avanzada de la Universidad Andrés Bello, ScientificElectronic Library Online (Scielo) y Literatura Latinoamericana y del Caribe en Ciencias de la Salud (Lilacs).

\section{Material Y Método}

De acuerdo a la importancia de la relación entre la satisfacción usuaria y la atención primaria de salud, es que se considera relevante una búsqueda de estudios relacionados a la pregunta de investigación planteada anteriormente, para conocer los factores que influyen en ella.

Los términos utilizados para realizar la búsqueda fueron "satisfacción usuaria", "calidad de atención", "primer nivel salud" y "atención primaria salud". Para acotar la búsqueda se definen criterios de inclusión tales como textos completos, idioma español, Latinoamericanos, publicados entre los años 2000 a 2016 y de acceso gratuito. Por otra parte, los criterios de exclusión fueron "segundo nivel" y "tercer nivel".

Los años fueron seleccionados en base a la transición del modelo de salud primaria desde un enfoque biomédico utilizado en los consultorios, hacia el modelo de atención con enfoque familiar en la atención primaria de salud, implementado en los centros de salud familiar en el año 1997 y que continúa vigente. Se utilizó una base de datos gratuita para los investigadores, como los recursos electrónicos con búsqueda avanzada de la Universidad Andrés Bello, la cual contiene acceso liberado a los textos que cumplieron con los criterios seleccionados. Asimismo, es posible encontrarlos indexados en Scielo, Lilacs, Medline y EBSCO y de esta forma asegurar la obtención de información confiable y respaldada. La búsqueda se realizó entre los meses de Octubre -Noviembre del año 2016, a través de 
las bases mencionadas, donde en primer lugar mediante multibuscador se obtuvieron 74 artículos, posteriormente se eliminaron por repetición 32, quedando un total de 42 artículos, de los cuales se seleccionaron 5. Para complementar la búsqueda, se ingresó directamente a la base de datos de ScientificElectronic Library Online y Literatura Latinoamericana y del Caribe en Ciencias de la Salud, encontrando 7 estudios adicionales que cumplían con los criterios de inclusión y el resto fueron descartados. Es así como finalmente se analizaron 12 artículos de investigación que respondieron al objetivo del estudio.

\section{Resultados}

En respuesta a la interrogante investigativa y en relación a los criterios de inclusión, la Tabla 1 muestra los 12 artículos escogidos. Donde 5 de ellos se encontraron en la base de datos de la Universidad Andrés Bello y 7 en ScientificElectronic Library Online y Literatura Latinoamericana y del Caribe en Ciencias de la Salud. Son señalados también los autores de referencia, país de realización de los estudios, años de publicación que comprenden desde el 2003 al 2016, e indexación como referencia de búsqueda, favoreciendo su consulta y análisis (Tabla1).

Tabla $\mathrm{N}^{0}$ 1: Artículos seleccionados de acuerdo a los criterios de inclusión, Chile 2016.

\begin{tabular}{|c|c|c|c|c|}
\hline Artículos & $\begin{array}{l}\text { Año, } \\
\text { País }\end{array}$ & Autor & Métodos y objetivo & Variables estudiadas \\
\hline $\begin{array}{l}\text { Satisfacción usuaria: Un } \\
\text { indicador de calidad del } \\
\text { Modelo de Salud }^{(5)} \text {. }\end{array}$ & $\begin{array}{l}2004, \\
\text { Chile }\end{array}$ & $\begin{array}{ll}\text { Carolina } & \text { Oliva; } \\
\text { Carmen } & \text { Gloria } \\
\text { Hidalgo. } & \\
\end{array}$ & $\begin{array}{l}\text { Estudio cuasi experimental, de } \\
\text { mediciones repetidas pre-post } \\
\text { intervención de salud, el cual } \\
\text { evaluó la calidad total del } \\
\text { programa de familias en riesgo } \\
\text { biopsicosocial (PRODEFA II) } \\
\text { comparado con los programas } \\
\text { tradicionales de la AP. }\end{array}$ & $\begin{array}{l}\text { Las variables se } \\
\text { organizaron según las } \\
\text { dimensiones de estructura, } \\
\text { proceso y resultado de la } \\
\text { atención. }\end{array}$ \\
\hline $\begin{array}{l}\text { Desarrollo de un } \\
\text { indicador de la calidad } \\
\text { de atención en Centros } \\
\text { de Salud Familiar para la } \\
\text { medición de la eficiencia } \\
\text { técnica }{ }^{(6) .}\end{array}$ & $\begin{array}{l}2015, \\
\text { Chile }\end{array}$ & $\begin{array}{l}\text { Martha Ramírez- } \\
\text { Valdivia; Jennifer } \\
\text { Lorena Mendoza- } \\
\text { Alonzo; Ana } \\
\text { Fabiola Moraga- } \\
\text { Pumarino }\end{array}$ & $\begin{array}{l}\text { Estudio descriptivo-explicativo, } \\
\text { cuyo objetivo fue desarrollar y } \\
\text { validar un indicador que mide la } \\
\text { calidad del servicio en la } \\
\text { Atención Primaria de Salud. }\end{array}$ & $\begin{array}{l}\text { Se analizaron dos } \\
\text { elementos: estructura y } \\
\text { proceso, definidos } \\
\text { por cuatro dimensiones: } \\
\text { recurso humano, factor } \\
\text { capital, extensión de uso y } \\
\text { resolutividad }\end{array}$ \\
\hline $\begin{array}{l}\text { Evaluación de la } \\
\text { satisfacción usuaria en } \\
\text { un Centro de Atención } \\
\text { Primaria de Salud }{ }^{(7)} \text {. }\end{array}$ & $\begin{array}{l}\text { 2013, } \\
\text { Chile }\end{array}$ & $\begin{array}{l}\text { María Javiera } \\
\text { Pérez V.; Lucy } \\
\text { Aceituno. }\end{array}$ & $\begin{array}{l}\text { Estudio descriptivo transversal. } \\
\text { El objetivo fue evaluar la } \\
\text { percepción de los usuarios del } \\
\text { CESFAM Puertas Negras hacia } \\
\text { la atención de Salud, como } \\
\text { también, el grado de } \\
\text { satisfacción usuaria frente a la } \\
\text { atención de los distintos } \\
\text { funcionarios }\end{array}$ & $\begin{array}{l}\text { Los aspectos evaluados } \\
\text { fueron: acceso a la } \\
\text { atención, calidad de la } \\
\text { atención, recomendación a } \\
\text { otros usuarios, } \\
\text { expectativas del usuario y } \\
\text { satisfacción global. }\end{array}$ \\
\hline $\begin{array}{l}\text { Evaluación } \\
\text { cumplimiento de los } \\
\text { atributos de la Atención } \\
\text { Primaria y grado de } \\
\text { satisfacción de los } \\
\text { usuarios de de un } \\
\text { establecimiento de de } \\
\text { primer nivel de atención } \\
\text { (8). }\end{array}$ & $\begin{array}{l}\text { 2011, } \\
\text { Perú }\end{array}$ & $\begin{array}{lr}\text { María } & \text { Cuba- } \\
\text { Fuentes; } & \text { Arturo } \\
\text { Jurado; } & \text { Eva } \\
\text { Estrella. } & \end{array}$ & $\begin{array}{l}\text { Estudio descriptivo transversal } \\
\text { cuyo objetivo fue evaluar el } \\
\text { cumplimiento de la estrategia } \\
\text { de atención primaria y el grado } \\
\text { de satisfacción de los usuarios } \\
\text { en un establecimiento de salud } \\
\text { de primer nivel }\end{array}$ & $\begin{array}{l}\text { Se midió el grado de } \\
\text { satisfacción con respecto a } \\
\text { la estructura, los procesos } \\
\text { y los resultados del } \\
\text { establecimiento, y el } \\
\text { cumplimiento de tres } \\
\text { atributos de la atención } \\
\text { primaria: primer contacto, } \\
\text { integralidad y continuidad. }\end{array}$ \\
\hline $\begin{array}{l}\text { Satisfacción sobre la } \\
\text { infraestructura y la } \\
\text { calidad de atención en la } \\
\text { consulta de Gineco- } \\
\text { Obstetricia de un } \\
\text { establecimiento } \\
\text { atención primaria }^{(9)} \text { de }\end{array}$ & $\begin{array}{l}\text { 2016, } \\
\text { Perú }\end{array}$ & $\begin{array}{l}\text { Sara Zamora- } \\
\text { Chávez. }\end{array}$ & $\begin{array}{l}\text { Se realizó un estudio } \\
\text { observacional no experimental } \\
\text { en el servicio de Gineco } \\
\text { Obstetricia del Hospital de la } \\
\text { Solidaridad Comas de Lima, } \\
\text { utilizando el instrumento } \\
\text { SERVQUAL modificado. El } \\
\text { objetivo fue determinar el grado } \\
\text { de satisfacción de los usuarios } \\
\text { externos acerca de la } \\
\text { infraestructura y la calidad de } \\
\text { atención }\end{array}$ & $\begin{array}{l}\text { Se analizaron las } \\
\text { dimensiones de: Respeto } \\
\text { al usuario, Eficacia, } \\
\text { Información completa, } \\
\text { Accesibilidad, } \\
\text { Infraestructura } \\
\text { Seguridad, y Satisfacción } \\
\text { global: }\end{array}$ \\
\hline
\end{tabular}




\begin{tabular}{|c|c|c|c|c|}
\hline $\begin{array}{l}\text { Satisfacción de usuarias } \\
\text { del control prenatal en } \\
\text { instituciones de salud } \\
\text { pública y } \\
\text { asociados. } \\
\text { (10). }\end{array}$ & $\begin{array}{l}\text { 2014, } \\
\text { Colombia }\end{array}$ & $\begin{array}{|lr|}\text { Irma } & \text { Castillo- } \\
\text { Ávila; } & \text { María } \\
\text { Villareal- } & \\
\text { Villanueva; } & \\
\text { Elizabeth } & \text { Olivera- } \\
\text { Correa; } & \text { Alba } \\
\text { Pinzón- } & \end{array}$ & $\begin{array}{l}\text { Se realizó un estudio analítico, } \\
\text { en } 25 \text { instituciones públicas de } \\
\text { Cartagena utilizando la } \\
\text { "Encuesta de satisfacción de } \\
\text { usuarios externos de los } \\
\text { servicios ambulatorios en } \\
\text { Instituciones Prestadoras de }\end{array}$ & $\begin{array}{l}\text { Se evaluaron seis } \\
\text { dimensiones que incluyen: } \\
\text { derechos y deberes, } \\
\text { registro e ingreso, } \\
\text { evaluación de necesidades } \\
\text { al ingreso, planeación y } \\
\text { ejecución del cuidado y }\end{array}$ \\
\hline & & $\begin{array}{l}\text { Consuegra; } \\
\text { Heidy Carrascal- } \\
\text { Soto. }\end{array}$ & $\begin{array}{l}\text { Servicios de Salud IPS". El } \\
\text { objetivo fue determinar los } \\
\text { factores asociados a la } \\
\text { satisfacción de usuarias de } \\
\text { servicios de control } \\
\text { prenatal en instituciones de } \\
\text { salud de primer nivel de la red } \\
\text { pública de Cartagena }\end{array}$ & $\begin{array}{l}\text { tratamiento, salida y } \\
\text { seguimiento y percepción } \\
\text { sobre ambiente físico de la } \\
\text { institución. Además, se } \\
\text { midió el nivel de } \\
\text { satisfacción global. }\end{array}$ \\
\hline $\begin{array}{l}\text { Atención prenatal en el } \\
\text { primer nivel de atención: } \\
\text { características de los } \\
\text { proveedores que influyen } \\
\text { en la satisfacción de las } \\
\text { usuarias }{ }^{(11)} \text {. }\end{array}$ & $\begin{array}{l}\text { 2003, } \\
\text { México }\end{array}$ & $\begin{array}{l}\text { Mario Bronfman- } \\
\text { Pertzovsky; } \\
\text { Sergio López- } \\
\text { Moreno; Carlos } \\
\text { Magis-Rodríguez; } \\
\text { Alejandra } \\
\text { Moreno- } \\
\text { Altamirano; } \\
\text { SheaRutstein. }\end{array}$ & $\begin{array}{l}\text { Estudio descriptivo transversal } \\
\text { cuyo objetivo fue evaluar el } \\
\text { nivel de satisfacción alcanzado } \\
\text { por las usuarias de los servicios } \\
\text { de atención prenatal en el } \\
\text { primer nivel de atención en } \\
\text { México, y comparar con } \\
\text { algunas características del } \\
\text { proveedor y del servicio. }\end{array}$ & $\begin{array}{l}\text { Se estudiaron los procesos } \\
\text { de atención, trato recibido } \\
\text { en la consulta médica, en } \\
\text { este caso se consideraron } \\
\text { los dominios de: a) calidad } \\
\text { técnica y b) calidad } \\
\text { interpersonal y contextual. }\end{array}$ \\
\hline $\begin{array}{llr}\text { Descripción } & \text { de } & \text { la } \\
\text { satisfacción } & \text { usuaria } & \text { de } \\
\text { pacientes } & \text { que } & \text { reciben } \\
\text { atención de } & \text { urgencia } \\
\text { dental en } & \text { cinco } \\
\text { establecimientos } & \text { de } \\
\text { atención } & \text { primaria } & \text { de } \\
\text { salud }^{(12)} \text {. } & & \end{array}$ & $\begin{array}{l}\text { 2012, } \\
\text { Chile }\end{array}$ & $\begin{array}{l}\text { Cristina Bucchi; } \\
\text { Constanza } \\
\text { Sepúlveda; María } \\
\text { Monsalves; Luis } \\
\text { Bustos. }\end{array}$ & $\begin{array}{l}\text { Estudio de corte transversal. Se } \\
\text { planteó conocer el porcentaje } \\
\text { de satisfacción usuaria de } \\
\text { pacientes que reciben atención } \\
\text { de urgencia dental en cinco } \\
\text { establecimientos de Atención } \\
\text { primaria de la salud de la } \\
\text { ciudad de Temuco. }\end{array}$ & $\begin{array}{l}\text { Se analizaron las } \\
\text { siguientes variables: } \\
\text { Urgencia dental, tiempo de } \\
\text { espera, horario de } \\
\text { atención, trato del } \\
\text { profesional, tiempo de } \\
\text { atención, explicación del } \\
\text { profesional, equipamiento } \\
\text { del establecimiento, } \\
\text { dotación, resolución del } \\
\text { problema y satisfacción } \\
\text { general. }\end{array}$ \\
\hline $\begin{array}{l}\text { Satisfacción de } \\
\text { pacientes que acudieron } \\
\text { al primer nivel de } \\
\text { atención en Bogotá }{ }^{(13)} \text {. }\end{array}$ & $\begin{array}{l}2014, \\
\text { Colombia }\end{array}$ & $\begin{array}{l}\text { Juan González- } \\
\text { Quiñones; } \\
\text { Guillermo } \\
\text { Restrepo- } \\
\text { Chavarriaga; } \\
\text { Astrid } \\
\text { Hernández- } \\
\text { Rojas; Diana } \\
\text { Ternera- } \\
\text { Saavedra; Camilo } \\
\text { Galvis-Gómez; } \\
\text { Jenny Pinzón- } \\
\text { Ramírez. }\end{array}$ & $\begin{array}{l}\text { Tipo de estudio: descriptivo de } \\
\text { tipo corte transversal. El } \\
\text { objetivo fue estimar la } \\
\text { percepción de los pacientes } \\
\text { sobre la atención médica en el } \\
\text { primer nivel de atención. }\end{array}$ & $\begin{array}{l}\text { Se estudiaron las } \\
\text { siguientes variables: } \\
\text { relaciones } \\
\text { médico-paciente y acerca } \\
\text { del proceso de atención } \\
\text { médica. }\end{array}$ \\
\hline $\begin{array}{l}\text { Calidad de atención y } \\
\text { satisfacción del usuario } \\
\text { con cita previa en } \\
\text { medicina familiar de } \\
\text { Guadalajara }{ }^{(14)} \text {. }\end{array}$ & $\begin{array}{l}2007, \\
\text { México }\end{array}$ & $\begin{array}{l}\text { Cecilia Colunga- } \\
\text { Rodríguez; Marco } \\
\text { López-Montoya; } \\
\text { Guadalupe } \\
\text { Aguayo-Alcaraz; } \\
\text { José Canales- } \\
\text { Muñoz. }\end{array}$ & $\begin{array}{l}\text { Estudio de diseño transversal } \\
\text { analítico cuyo objetivo fue } \\
\text { evaluar la calidad de la atención } \\
\text { y satisfacción del usuario con } \\
\text { cita previa en unidades de } \\
\text { medicina familiar. }\end{array}$ & $\begin{array}{l}\text { Se exploró calidad del } \\
\text { servicio y satisfacción del } \\
\text { usuario. En calidad se } \\
\text { evaluaron } 7 \text { dimensiones: } \\
\text { oportunidad de la atención, } \\
\text { amabilidad del personal, } \\
\text { proceso de la atención, } \\
\text { resultados de la atención, } \\
\text { atención personalizada, } \\
\text { suficiencia de la } \\
\text { información y comodidad } \\
\text { de las instalaciones. En } \\
\text { satisfacción usuaria se } \\
\text { evaluó al médico familiar, } \\
\text { Unidades de Medicina } \\
\text { Familiar y otros servicios. }\end{array}$ \\
\hline $\begin{array}{lr}\text { Calidad de } & \text { servicio: } \\
\text { satisfacción } & \text { usuaria } \\
\text { desde la perspectiva de } \\
\text { enfermeria }{ }^{(15)} \text {. }\end{array}$ & $\begin{array}{l}2014, \\
\text { Chile }\end{array}$ & $\begin{array}{l}\text { Amanda } \\
\text { Gallardo-Ferrada, } \\
\text { Katiuska } \\
\text { Reynaldos- } \\
\text { Grandón. }\end{array}$ & $\begin{array}{l}\text { Revisión sistemática cuyo } \\
\text { objetivo fue analizar los } \\
\text { estudios realizados en el área } \\
\text { de salud con enfoque en } \\
\text { percepción y expectativas de } \\
\text { servicio. }\end{array}$ & $\begin{array}{lr}\text { Todos los artículos } \\
\text { comprendieron } & \text { la } \\
\text { evaluación de la } \\
\text { percepción y la calidad de } \\
\text { servicio. }\end{array}$ \\
\hline 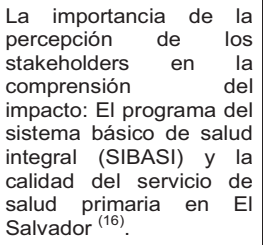 & $\begin{array}{l}2007 \\
\text { El } \\
\text { Salvador }\end{array}$ & Jill Murphy. & $\begin{array}{l}\text { Investigación cuali-cuantitativa } \\
\text { cuyo objetivo fue conocer las } \\
\text { percepciones de diferentes } \\
\text { actores sobre el impacto inicial } \\
\text { del programa sibasien la } \\
\text { calidad del servicio de salud. }\end{array}$ & $\begin{array}{l}\text { Las variables para medir el } \\
\text { impacto del programa } \\
\text { Sibasi (Sistema Básico de } \\
\text { Salud Integral) en la } \\
\text { calidad de la atención } \\
\text { fueron: participación, } \\
\text { acceso y calidad en salud } \\
\text { y percepciones de varios } \\
\text { stakeholders. }\end{array}$ \\
\hline
\end{tabular}


Al revisar los artículos seleccionados, la investigación "Desarrollo de un indicador de la calidad de atención en Centros de Salud Familiar para la medición de la eficiencia técnica" (6), estudio descriptivo explicativo realizado en Chile, tuvo como objetivo crear y validar un indicador que midiera la calidad del servicio en la Atención Primaria de Salud -APS- en lo que a eficiencia técnica se refiere. La muestra fue el personal que trabaja en APS con responsabilidad directiva para validar el indicador y luego se trabajó con cuatro centros de salud familiar de la ciudad de Temuco para su aplicación y estimación de valores. El indicador se valoró según su estructura y proceso, no así el elemento resultado, debido a las características de la APS. Las dimensiones de estructura a evaluar incluyen subindicadores como dotación del equipo de cabecera, del equipo transversal, capacitaciones al equipo de salud, espacio físico, y vehículos para traslado. Estos subindicadores se consideraron para mejorar la eficiencia de las prestaciones y el acceso de la población, hacer que el equipo de salud sea más resolutivo y permitir la realización de actividades con una adecuada capacidad de planta física. Por otra parte, las dimensiones de proceso contenían la extensión de uso tanto de actividades de promoción, prevención como de consultas por morbilidad y la derivación a la red asistencial. Se obtuvo una ponderación de un $40 \%$ en la estructura y un $60 \%$ para el proceso, puesto que la interacción del paciente con el equipo de salud es primordial para la satisfacción que pueda tener el usuario luego de su atención de salud. Se recomienda establecer estándares de comparación para los subindicadores y estudios que estimen el valor del indicador a nivel nacional.

$\mathrm{Al}$ analizar el estudio "Evaluación de la satisfacción usuaria en un Centro de Atención Primaria de Salud" (7), realizado en el CESFAM Puertas Negras de Chile, en donde se aplicó un cuestionario a 252 usuarios, el cual consistió en 12 preguntas, las que consideraron los siguientes aspectos: Acceso a la atención, calidad de la atención, recomendación a otros usuarios, expectativas del usuario y satisfacción global, de estos, el punto peor evaluado fue el acceso de los usuarios a la atención, especialmente en los que se refiere a la facilidad de los usuarios para contactar el centro por teléfono y a la facilidad para conseguir las horas de atención. Otros de los puntos claves fueron en relación a la puntualidad de la atención médica. La impuntualidad en la atención está relacionada en que los tiempos de atención son reducidos y para poder entregar una atención mínimamente satisfactoria, se hace imposible respetar estos tiempos. Lo anterior, junto a la alta rotación de funcionarios que existe en el centro, influye en gran medida en que la satisfacción usuaria sea deficiente. Se debe destacar que en el ítem calidad de la encuesta no se consideraron aspectos relacionados con la calidad técnico-científica de los prestadores de salud, por lo que podría dificultar las posibilidades de perfeccionamiento de los funcionarios producto de la alta demanda asistencial.

En Perú se publicó el estudio "Evaluación del cumplimiento de los atributos de la Atención Primaria y grado de satisfacción de los usuarios de un establecimiento de primer nivel de atención "(8), estudio descriptivo transversal cuyo objetivo fue evaluar el cumplimiento de la estrategia de atención primaria y el grado de satisfacción de los usuarios en un establecimiento de salud de primer nivel. Este estudio se efectuó en el Policlínico Juan José Rodríguez Lazo, un establecimiento de atención primaria de Seguridad Social del Perú (EsSalud), a través de 2 encuestas que fueron validadas en un estudio piloto, una encuesta que buscó medir satisfacción con el servicio de salud y otra sobre los atributos de la atención primaria. Estas fueron aplicadas a 240 participantes y evaluaron la satisfacción de los usuarios de establecimientos de salud, considerando 9 parámetros: horario del establecimiento, tiempo de espera en la consulta, tiempo de espera en emergencia, atención por el médico, enfermera y odontólogo en la consulta, resolutividad, infraestructura del establecimiento, exámenes auxiliares, medicación prescrita y accesibilidad geográfica, obteniendo como resultado un grado de satisfacción medio. La evaluación de los tres atributos básicos de la atención primaria consideró; primer contacto, integralidad y continuidad en el servicio de salud. Los resultados de la evaluación de los atributos 
en general, arrojaron que el 57,1\% de los encuestados percibió el cumplimiento como medio, mientras que $30 \%$ percibió que nunca se cumplían. Por separado, la evaluación de primer contacto mostró que los usuarios refirieron un cumplimiento medio, en continuidad se obtuvo el mismo resultado y en integralidad el grado de cumplimiento fue bajo. Finalmente, el resultado de satisfacción medio de parte de los usuarios coincide con un cumplimiento medio de los atributos de la atención primaria, aunque no se puede establecer una asociación. Como estrategia en atención primaria es necesario determinar el logro de las características esenciales para la consecución de sistemas más costo-efectivos y equitativos.

El artículo "Satisfacción Usuaria: Un Indicador de Calidad del Modelo de Salud Familiar, Evaluada en un Programa de Atención de Familias en Riesgo Biopsicosocial, en la Atención Primaria"(5), realizado en Chile, es un análisis comparativo de la satisfacción usuaria entre los consultantes del Programa de Familias (PRODEFA II) y los usuarios de programas tradicionales, del mismo centro de salud de atención primaria. El grupo experimental y de control se conformó con 2500 familias en situación de pobreza, de las cuales se obtuvo una muestra de 139 familias según un perfil de riesgo biopsicosocial alto y moderado, que consultaron durante un año en el Centro de Salud Villa O'Higgins, en la comuna de la Florida en la Región Metropolitana. A este grupo se les aplicó una Encuesta de Satisfacción usuaria (ESU), la cual considera tres dimensiones de la calidad de la atención, siendo estos: estructura, proceso y resultado. Los resultados obtenidos permiten afirmar que se encontró un mayor nivel de satisfacción usuaria por parte del grupo experimental, respecto al estado de salud actual comparado con los últimos seis meses, mayor adherencia a los tratamientos, mayor resolutividad con los pacientes crónicos y una mejor evaluación general de la atención de salud. Los análisisestadísticosindicaron que los usuarios del programa de salud familiar evalúan significativamente mejor la atención recibida en comparación a los usuarios de programas tradicionales, por lo que se determina que un programa basado en un modelo biopsicosocial de atención, genera mayor satisfacción en los usuarios, a diferencia de los programas tradicionales basados en un modelo exclusivamente biomédico.

También en Perú, se realizó una investigación referida a: "Satisfacción sobre la infraestructura y la calidad de atención en la consulta de Gineco-Obstetricia de un establecimiento de atención primaria"(()), tiene una metodología de tipo cuantitativa, cuyo fin fue determinar el grado de satisfacción de los usuarios externos acerca de la infraestructura y de la calidad de atención en la consulta externa del servicio de ginecoobstetricia del Hospital de la Solidaridad de Comas. El tamaño muestral para el estudio corresponde a un total de 249 encuestas, las que incluyen 16 preguntas distribuidas en dos partes. La primera corresponde a los aspectos generales y la segunda es referida a la percepción del usuario externo respecto a la atención recibida. Las preguntas abordan las siguientes temáticas: respeto al usuario, eficacia, cuidado y atención individualizada, información completa, accesibilidad, satisfacción global, infraestructura y seguridad.Se concluye a modo de resultado que el grado de satisfacción acerca de la infraestructura y de la calidad de atencióncorresponde a un $70,92 \%$, por otra parte, el grado de satisfacción corresponde a un 87,55\%.Finalmente, la satisfacción usuaria depende de la calidad brindada por los servicios de salud, así como de las expectativas que este posee.

En Colombia, la investigación "Satisfacción de usuarias del control prenatal en instituciones de salud públicas y factores asociados. Cartagena"(10), es un estudio analítico, con diseño de encuesta, con una muestra de 712 embarazadas de bajo riesgo atendidas en 25 instituciones públicas de Cartagena.Para la recolección de información se utilizó la "Encuesta de satisfacción de usuarios externos de los servicios ambulatorios en Instituciones Prestadoras de Servicios de Salud IPS", la cual evaluó seis dimensiones: derechos y deberes, registro e ingreso, evaluación de necesidades al ingreso, planeación y ejecución del cuidado y tratamiento, salida y seguimiento y percepción sobre ambiente 
físico de la institución. Además, presenta una subescala que mide el nivel de satisfacción global. El 84\% de las encuestadas manifestaron sentirse satisfechas con el servicio que recibieron en la IPS donde fueron atendidas. Los factores implícitos en las dimensiones evaluadas que explica la satisfacción de las usuarias del servicio de control prenatal fueron: proceder del área urbana y recibir buen trato por parte del personal de enfermería. En cuanto a la relación que existe entre el nivel de escolaridad y la satisfacción de las usuarias, se encontró que aquellas gestantes que han cursado estudios superiores estaban más satisfechas con la información recibida sobre sus derechos y deberes, el tiempo de espera y el servicio directo de la oficina de atención que aquellas cuya formación académica era inferior.

En México, el estudio “Atención prenatal en el primer nivel de atención: características de los proveedores que influyen en la satisfacción de las usuarias"(11), tiene por objetivo, evaluar el nivel de satisfacción alcanzado por las usuarias de los servicios de atención prenatal en el primer nivel de atención en México. La metodología usada fue una encuesta epidemiológica en la que se utilizó una muestra de 95 centros de salud pertenecientes a la secretaría de salud, en la cual se analizó la relación existente entre la capacidad de resolución clínica de los proveedores, el trato recibido durante la consulta y la satisfacción sobre la misma que la usuaria manifestó en entrevista directa.Esta investigación muestra que los servicios de atención prenatal de primer nivel, resultan insatisfactorios para las usuarias con menores recursos económicos y que en general esperan más tiempo para recibir la atención. Se observa además una tendencia a recibir peor trato durante la consulta conforme es menor el nivel socioeconómico y escolaridad. La comodidad, confidencialidad y privacidad son considerados factores importantes. Como propuesta de mejora, son necesarias las evaluaciones de satisfacción usuaria en relación con la atención recibida especialmente en las zonas más vulnerables.

En el sur de Chile, la publicación "Descripción de la satisfacción usuaria de pacientes que reciben atención de urgencia dental en cinco establecimientos de Atención Primaria de Salud"(12), estudio de corte transversal, realizado en la ciudad de Temuco. Suobjetivo fue conocer el porcentaje de satisfacción usuaria de pacientes que reciben atención de urgencia dental en cinco establecimientos de Atención Primaria de Salud -APS-, por ende, se aplicó un formulario estandarizado a 305 personas seleccionadas en forma aleatoria, determinando que el $90 \%$ de los pacientes encuestados relata estar satisfecho con la atención recibida, mientras que el 9,5\% dice no estar satisfecho con la atención otorgada.De los parámetros mejor evaluados se encuentra el trato al paciente con un $99 \%$ y la información entregada por parte del dentista sobre los medicamentos con un 96,8\%, mientras que los parámetros peor evaluados son la opinión del paciente en relación a la cantidad de personal dental, donde un $64,5 \%$ considera que no existen suficientes dentistas para abastecer la demanda y el tiempo de espera considerado excesivo en un $4,9 \%$ de los casos. Debido a la escasez de estudios relacionados a la satisfacción usuaria en Chile, se sugiere seguir una búsqueda investigativa que incluya una escala de medición exacta, que fundamente un plan de mejora.

También en Colombia, la evidencia de "Satisfacción de pacientes que acudieron al primer nivel de atención en Bogotá" (13), de tipo descriptivo de corte transversal tuvo como objetivo estimar la percepción de los pacientes sobre la atención médica en el primer nivel de atención. Para llevar a cabo la investigación se realizó una encuesta telefónica a 804 personas pasado un mes de la cita médica, que fueran mayores de 15 años y aceptaran participar.El estudio midió variables generales como; características demográficas, relación médico-paciente sobre el proceso de comunicación, proceso de atención médica en relación a la continuidad en la atención y percepción del acto médico. Las sub-variables arrojaron que el tiempo promedio de acceso a la atención fue de 9,6 días, el 78 \% refiere haber podido contar todo lo que sentía al médico, $30 \%$ sintió alivio completo luego de la atención médica, el médico explicó en un $60 \%$ de los casos el diagnóstico y uno de cuatro 
indagó en aspectos familiares.Concluyendo entonces, lo que un paciente quiere cuando es atendido por el médico es ser escuchado, rescatando las relaciones humanas, dejando en manifiesto que la comunicación extensa con el paciente es tan importante como el enfrentar la enfermedad en el acto médico.

En relación al artículo "Calidad de atención y satisfacción del usuario con cita previa en medicina familiar de Guadalajara"(14), realizado en México, cuyo objetivo fue evaluar la calidad de la atención y satisfacción del usuario con cita previa en unidades de medicina familiar a través de un estudio con diseño transversal analítico, trabajó con una muestra probabilística de 983 usuarios caracterizada por un $67 \%$ de sexo femenino, edad 40,83 $\pm 21,6$ años, $40 \%$ asalariados, $67 \%$ casados, tiempo de espera 55,46 $\pm 74,03$ min y $56 \%$ con cita previa. La investigación utilizó dos instrumentos validados; uno para evaluar la "Satisfacción del usuario" que consistió en un inventario que explora tres dimensiones: médico familiar, unidad de medicina familiar y otros servicios y otro para medir "Calidad de la atención" midiendo: oportunidad de la atención, amabilidad del personal, proceso de la atención, resultados de la atención, atención personalizada, suficiencia de la información y comodidad de las instalaciones.El resultado mostró que la calidad de la atención en las dimensiones mencionadas se calificó como alta, el tiempo de espera en dos de las tres unidades alcanzó el 36\% de calidad. La satisfacción del usuario en dimensión médico familiar fue de un $79 \%$, unidad de medicina familiar mostró calificación de un $59 \%$ y otros servicios calificaron con un 53\%. Finalmente, la calidad de atención fue alta, excepto en tiempo de espera, la satisfacción del usuario se asoció con la cita previa y en coincidencia con otros hallazgos, los usuarios adultos mayores se mostraron más satisfechos y calificaron más alta la calidad de la atención.

Es interesante analizar el artículo "Calidad de servicio: satisfacción usuaria desde la perspectiva de enfermería" (15), que es una revisión sistemática de datos que tuvo por objetivo analizar los estudios realizados en el área de salud con enfoque en percepción y expectativas de servicio, en el cual se seleccionaron 17 artículos permitiendo la inclusión simultánea de investigaciones cuantitativas (experimental y no experimental) y cualitativas con el fin de comprender mejor el fenómeno de interés, de tal modo se formuló la pregunta de investigación; ¿cómo condiciona la percepción y las expectativas del usuario la satisfacción en relación a los servicios de salud otorgados?.Como resultado se determinó que todos los artículos comprenden la evaluación de la percepción y la calidad de servicio y que, de la selección, 15 de ellos hacen referencia a servicios de salud. La calidad de servicio debe cumplir con ciertas dimensiones como son: la dimensión técnica referida a que el prestador cuente con conocimientos, habilidades para la realización de procedimientos clínicos adecuados y oportunos de conformidad con las necesidades del cliente, la dimensión interpersonal referida a la interacción entre prestador y cliente en la actividad de atención, respeto y cordialidad mutua, por último, la dimensión de infraestructura que evalúa las características del lugar como la limpieza, iluminación y ventilación del ambiente. Es importante destacar que el estudio evidencia que los resultados en la satisfacción variarán de acuerdo al instrumento de recolección de datos seleccionado para llevar a cabo el estudio, de ahí la importancia en la experticia y formación de las personas que incurran en esta área. Finalmente, la calidad de servicio es percibida de diferentes maneras y existen múltiples factores que determinan la percepción de calidad de servicio.

En El Salvador se realizó el estudio "La importancia de la percepción de los stakeholders en la comprensión del impacto: El programa del Sistema Básico de Salud Integral (SIBASI) y la calidad del servicio de salud primaria en El Salvador" (16), de tipo cualicuantitativo, que recoge los resultados de una evaluación preeliminar, del impacto del programa Sibasi sobre la calidad del servicio de salud a nivel primario en El Salvador, según la percepción de variados actores del programa, tales como: 1) formuladores de políticas o representantes institucionales (representantes de agencias internacionales 
de financiamiento y desarrollo); 2) miembros de la sociedad civil u otros expertos (representantes de las ONG de salud, doctores o trabajadores de la salud que laboran con sectores de las ONG y miembros y académicos); 3) administradores y personal de Sibasi (directores, subdirectores, y personal técnico de Sibasi), y 4) pacientes o usuarios potenciales/ miembros de la comunidad (miembros de la comunidad, técnicamente cubiertos por el sistema que, sin embargo, pueden o no utilizarlo). Las cuatro categorías de actores, perciben el programa de manera muy diferente, ya que por una parte hay grupos que son optimistas respecto del impacto y éxito del programa, otros se enfocan en los grandes problemas de cara al sistema de salud y la población salvadoreña. Por otro lado, se destacan continuos problemas de infraestructura, aunque el mayor problema de calidad detectado fue la disponibilidad de medicamentos. De forma concluyente se expresaron mejoras para lograr el éxito del programa en base a la calidad, para lo cual era necesario un plan de monitoreo que incluya diversidad regional, mayor inversión en salud por parte del gobierno, aspecto que limita la provisión de servicios de salud de calidad a la población.

\section{Discusión}

La interpretación de los datos obtenidos en los resultados indica que las publicaciones seleccionadas comprenden principalmente a los años 2007 - 2014, si bien uno de los criterios utilizados para la selección de estudios fue el periodo 2000 al 2016, no se encontraron publicaciones entre los años 2000 a 2002 según los criterios establecidos, sin embargo, fue posible encontrar estudios atingentes a la interrogante a investigar a partir del año 2003, 6 años después de la implementación del modelo de atención con enfoque familiar en la atención primaria de salud (1997), en Chile y la implementación de modelos biopsicosociales en salud apartando la visión biomédica a nivel mundial. Así mismo, el año de inicio de publicaciones que responden a la temática de este artículo, coincide con la resolución CD44.R6 del Consejo Directivo para el fortalecimiento de la atención primaria de salud, que culminó, además, con el compromiso de todos los gobiernos de las Américas para renovar la atención primaria de salud ${ }^{(1)}$.

En relación a los países oriundos, las publicaciones seleccionadas destacan mayoritariamente a; Chile y Colombia, justificado por los avances en los estudios sobre la atención de salud con enfoque humanizado, respeto a la dignidad del paciente y atención integral al paciente/usuario, familia y comunidad. La contribución de estos factores a la satisfacción usuaria resultan ser variables relevantes para la acreditación de instituciones y el aseguramiento de una atención de calidad.

Considerando los objetivos de los artículos, estos tienen relación con el conocer el grado o nivel de satisfacción en el primer nivel de atención de salud tanto por parte de los usuarios internos como externos, en diferentes contextos culturales y sociodemográficos.

Los resultados clarificados por el análisis de los estudios expuestos, concuerdan en su mayoría con la evaluación de los factores que influyen en la satisfacción usuaria como, el sexo, fluctuación de edad, estratificación socioeconómica, nivel educacional, tipo de atención, infraestructura del centro de salud, relación interpersonal paciente/usuario equipo de salud, tiempo de espera y acceso a la atención.

La satisfacción usuaria, por tanto, es un aspecto definitorio para la evaluación de la atención de calidad en salud, especialmente en el área de atención primaria pues es el inicio y primer acercamiento de la población a los sistemas de salud que abarcan un porcentaje considerable de población, a costos económicamente accesibles. Por tal motivo, resulta ser esencial el trabajo de los equipos de salud y los profesionales de enfermería en sí, para lograr niveles adecuados de atención que contribuyan a la satisfacción respondiendo a las necesidades y expectativas de quienes hagan uso de este nivel. Finalmente, los profesionales enfermeros en formación y en ejercicio activo de la profesión, deben iniciar un camino amplio a la investigación en Latinoamérica para sustentar estrategias efectivas, 
que logren contribuir en el tema abordado y aumentar la calidad de la atención.

\section{Conclusiones}

Respecto a los factores influyentes en la satisfacción usuaria de la atención primaria en salud Latinoamericana, es relevante mencionar que las investigaciones seleccionadas para la revisión integradora reúnen en amplia medida los factores que interfieren en el tema abordado, no obstante, cabe destacar que el conocer y estudiar el impacto de éstos no han podido dar respuesta a los aspecto de satisfacción en la atención de salud, pues a pesar de sugerir propuestas de mejoras no es posible visualizar cambios concretos en la evaluación del servicio entregado en el primer nivel de atención, siendo este el primer acceso y referencia a la salud.

Así como a nivel mundial se han hecho esfuerzos por fortalecer la atención primaria, para acercar a la comunidad a la promoción de salud y prevención de enfermedad, proporcionados por este nivel, se hace esencial impulsar el desarrollo de nuevos estudios que integren los factores influyentes en la satisfacción usuaria, no como un método de evaluación en sí, sino como un instrumento para crear las bases de mejoras en la atención de forma efectiva y considerando las necesidades de la población, con el objetivo de dar soluciones tanto a las expectativas como necesidades reales de los pacientes/usuarios. Esto hace fundamental el apoyo de los sistemas y entidades prestadoras de servicios de salud, para incorporar nuevas estrategias y ampliar la atención de forma estructurada tanto en salud/enfermedad como en aspectos del ámbito biopsicosocial. De forma concluyente se sugiere trabajar desde el respaldo de las investigaciones ya elaboradas para incentivar la creación de nuevos planes y lineamientos para una atención de calidad.

\section{Responsabilidades éticas:}

Confidencialidad de los datos: Los autores declaran que han seguido los protocolos de la Universidad Andrés Bello su centro de trabajo sobre el manejo confidencial de información. La investigación cuenta con la aprobación del Comité de Ética Científico de la Facultad de Enfermería de la Universidad.

\section{Bibliografía}

1 Macinko J, Montenegro H, Nebot Adell C, Etienne C y Grupo de Trabajo de Atención Primaria de Salud de la Organización Panamericana de la Salud. La renovación de la atención primaria de salud en las Américas. [Internet]. 2007. [citado el 23 de Oct. de 2016]; 21(2/3):73-84. Disponible en: http://www.scielosp.org/pdf/rpsp/v21n2-3/03.pdf

2 Organización Mundial de la Salud. Informe sobre la salud en el mundo 2008: La atención primaria de salud, más necesaria que nunca. [Internet]. Ginebra, Suiza: Organización Mundial de la Salud; 2008. [citado el 23 de Oct. de 2016]. Disponible en: http://www.who.int/whr/2008/08_report_es.pdf

3 Aguayo E, Rojas V. Manual de Apoyo a la Implementación del Modelo de Atención Integral con Enfoque Familiar y Comunitario. [Internet].Gobierno de Chile, Subsecretaría de Redes Asistenciales. [citado el 25 de Oct. de 2016]. Disponible en:http://www.bibliotecaminsal.cl/wp/wp-content/uploads/2016/03/18.pdf

4 Ministerio de Salud. Orientaciones para la implementación del modelo de atención integral de salud familiar y comunitaria. [Internet]. Gobierno de Chile, Subsecretaría de Redes Asistenciales. [citado el 26 de Oct. de 2016] Disponible en: http://web. minsal.cl/portal/url/item/e7b24eef3e5cb5d1e0400101650128e9.pdf

5 Oliva C, Hidalgo C. Satisfacción Usuaria: Un Indicador de Calidad del Modelo de Salud Familiar, Evaluada en un Programa de Atención de Familias en Riesgo Biopsicosocial, en la Atención Primaria. [Internet]. 2004 [citado el 23 de Oct. de 2016], 13(2); 173 - 186. Disponible en:http://www.scielo.cl/scielo.php?script=sci_arttext 
6 Ramírez M, Mendoza J, Moraga A. Desarrollo de un indicador de la calidad de atención en Centros de Salud Familiar para la medición de la eficiencia técnica [Internet]. 2015, Dic. [Citado el 27 de Nov. de 2016]; 33 (2): pp. 238-259. Disponible en: http://www.redalyc.org/articulo.oa?id=85241628006

7 Pérez M, Aceituno L. Evaluación de la satisfacción usuaria en un Centro de Atención Primaria de Salud [Internet]. 2013, Jun. [Citado el 28 de Nov. de 2016]; 53 (4): pp.233-240.Disponible en: http://pesquisa.bvsalud.org/portal/resource/pt/ lil-728138

8 Cuba-Fuentes M. Jurado A, Estrella E. Evaluación del cumplimiento de los atributos de la Atención Primaria y grado de satisfacción de los usuarios de un establecimiento de primer nivel de atención. [Internet]. 2011, Ene. [Citado el 28 de Nov.de 2016]; 22(1): pp.4-9. Disponible en: http://www.scielo.org.pe/scielo. php?script $=$ sci arttext\&pid $=$ S1018-130X2011000100002

9 Zamora S. Satisfacción sobre la infraestructura y la calidad de atención en la consulta de Gineco-Obstetricia de un establecimiento de atención primaria. [internet]. 2016, Feb. [Citado el 27 de Nov. de 2016]; 16 (1): pp 38-47. Disponible en: http://www. redalyc.org/articulo.oa?id $=371644760005$

10 Castillo I, Villarreal M, Olivera E, Pinzón A, Carrascal H. Satisfacción de usuarias del control prenatal en instituciones de salud públicas y factores asociados. Cartagena. [internet]. 2014, Mar. [Citado el 28 de Nov. de 2016];19(1): pp.128-140. Disponible en: http://www.scielo.org.co/pdf/hpsal/v19n1/v19n1a10.pdf

11 Bronfman-Pertzovsky M, López S, Magis C, Moreno A, Rutstein S. Atención prenatal en el primer nivel de atención: características de los proveedores que influyen en la satisfacción de las usuarias. [internet] 2003, Dic. [citado el 28 de Nov. de 2016] 45(6): pp.445-454.Disponible en: http://www.scielo.org.mx/scielo. php?script=sci arttext\&pid=S0036-36342003000600004\&lng=es.

12 Bucchi C, Sepúlveda C, Monsalves M, Bustos L. Descripción de la Satisfacción Usuaria de Pacientes que Reciben Atención de Urgencia Dental en Cinco Establecimientos de Atención Primaria de Salud.[Internet]. 2012. [citado el 28 de Nov. de 2016] 6(3): pp.275-280. Disponible en: http://www.scielo.cl/scielo. php?script=sci_arttext\&pid=S0718-381X2012000300005\&lng=es. http://dx.doi. org/10.4067/S0718-381X2012000300005

13 González-Quiñones J, Restrepo-Chavarriaga G, Hernández-Rojas A, TerneraSaavedra Galvis-Gómez C, Pinzón-Ramírez J. Satisfacción de pacientes que acudieron al primer nivel de atención en Bogotá. [Internet]. 2014, Mar. [citado el 28 de nov. de 2016]: 16 (6): pp. 871-884. Disponible en: http://www.scielosp.org/ scielo.php?pid $=$ S0124-00642014000600006\&script $=$ sci arttext

14 Colunga C, López M, Aguayo G, Canales J. Calidad de atención y satisfacción del usuario con cita previa en medicina familiar de Guadalajara. [Internet]. 2007, Ene. [citado el 28 de Nov. de 2016]: 33(3). Disponible en: http://www.redalyc.org/ articulo.oa? id $=21433312$

15 Gallardo-Ferrada A, Reynaldos-Grandón K. Calidad de servicio: satisfacción usuaria desde la perspectiva de enfermería. [Internet]. 2014, Oct. [citadoel 28 de Nov. de 2016]: 13(36): pp.353-363. Disponible en: http://scielo.isciii.es/scielo. php?script=sci arttext\&pid=S1695-61412014004400220\&lng=es\&nrm=iso.

16 Murphy J. La importancia de la percepción de los stakeholders en la comprensión del impacto: El programa del Sistema Básico de Salud Integral (SIBASI) y la calidad del servicio de salud primaria en El Salvador. Rev. Gerenc. Polit. Salud, Bogotá. [Internet]. 2007, Jun. [citado el 27 de Nov. de 2016]; 6 (12): pp. 31-50. Disponible en: http://rev_gerenc_polit_salud.javeriana.edu.co/vol6_n_12/dosier_2. pdf 